\title{
Modelagem e Implementação da Interface para Apresentação de Comportamentos Animados e Emotivos de um Agente Pedagógico Animado
}

\author{
Everton Bocca, Patrícia Jaques, Rosa Vicari \\ \{bocca, pjaques, rosa\}@inf.ufrgs.br \\ Instituto de Informática - PPGC - UFRGS
}

\begin{abstract}
This work presents an animated pedagogical agent's architecture for showing animated and emotive behavior, as the appearance of the character of this agent, which we calls PAT. This architecture was implemented in Java and JavaScript and Microsoft Agent. In order to define the agent's appearance it were made interviews with pedagogues, psychologists and designers.
\end{abstract}

Keywords: Multi-agent Systems, Distance Education, Animated Pedagogical Agents and Animated Agents

\section{Introdução}

Este trabalho encontra-se inserido no ambiente colaborativo suportado por computador, chamado MACES (Multiagent Architecture for an Collaborative Educational System), definido no projeto "Uma Proposta de Modelo Computacional de Aprendizagem à Distância Baseada na Concepção Sócio-Interacionista de Vygotsky" [AND 2000] [AND 2001]. Neste projeto, encontram-se em andamento duas teses de doutorado (Patrícia A. Jaques, Adja F. de Andrade), duas dissertações de mestrado (João L. Jung e Everton W. Bocca, já concluídas), juntamente com a colaboração de professores do grupo de Inteligência Artificial do Instituto de Informática da UFRGS (Prof. Rafael H. Bordini e Prof.a. Rosa M. Vicari).

A principal proposta deste trabalho é desenvolver e implementar a interface do personagem para o agente pedagógico animado Mediador, que opera no ambiente de aprendizado colaborativo MACES. O personagem desenvolvido chama-se PAT (Pedagogical and Affective Tutor).

A interface do personagem foi desenvolvida em Java, JavaScript e usa o Microsoft Agent [MIC 2003] para a apresentação das animações. Para definir a aparência do personagem foram feitas entrevistas com pedagogos, psicólogos, psicopedagogos e designers.

Na seção 2 apresentamos os conceitos que fazem parte da fundamentação teórica deste trabalho e que são necessários para o seu melhor entendimento. Na seção 3, apresentamos o ambiente educacional colaborativo, chamado MACES, na qual o agente pedagógico animado, que possui a interface definida neste trabalho, está inserido. $\mathrm{Na}$ seção 4, apresentamos a arquitetura do personagem para a apresentação dos 
comportamentos, bem como a metodologia usada para a definição da aparência do personagem. Finalmente, na seção 5, apresentamos as conclusões e na seção 6 as referências bibliográficas usadas neste trabalho.

\section{Fundamentação teórica}

O personagem animado proposto neste trabalho é a interface de um agente pedagógico animado que está inserido em um sistema educacional colaborativo à distância, chamado MACES. Este sistema foi modelado segundo a abordagem multiagente, onde cada agente deste ambiente possui uma função específica e interagem entre si a fim de realizar um objetivo global que é ensinar o aluno. Desta maneira, apresentamos nesta seção, os conceitos que identificamos necessários para o entendimento desta proposta: sistemas multiagentes (seção 0), agentes (seção 0) e agentes pedagógicos animados (seção 0 ).

\section{Sistemas Multiagentes (SMA)}

Um SMA pode ser definido como uma rede de resolvedores de problema que trabalham juntos para solucionar problemas que estão além das capacidades individuais (ou conhecimentos individuais) de cada resolvedor de problema [JEN 98]. Estes resolvedores de problemas (agentes) são autônomos e podem ser de natureza heterogênea. As características de um SMA são, conforme Jennings [JEN 98]:

$\sqrt{ }$ cada agente tem informação incompleta, ou capacidade para resolver o problema, assim, cada agente tem um ponto de vista limitado;

$\sqrt{ }$ não há nenhum controle de sistema global;

$\sqrt{ }$ dados descentralizados; $\mathrm{e}$

$\sqrt{ }$ computação é assíncrona.

A tecnologia de agentes pode trazer significativo suporte à aprendizagem na Web. As características de autonomia, colaboração e aprendizagem podem auxiliar na construção de um modelo de aprendiz e auxiliar na interação entre os alunos, incentivando o seu desenvolvimento sócio-cognitivo [GIR 99].

\section{Agentes}

Segundo Giraffa [GIR 99], o termo "agente" é usado com freqüência na IA, mas não há um consenso para o que um agente é ou significa e o classifica como sendo desde simples processos de hardware e/ou software até entidades sofisticadas com capacidade de realizar tarefas complexas. Mais especificamente, "agente" pode ser entendido como uma entidade que exibe alguns aspectos da inteligência humana.

Segundo Jennings [JEN 98], um agente é um sistema de computador, situado em algum ambiente que é capaz de uma ação autônoma flexível para atingir seus objetivos de projeto.

Segundo Sichman et al. [SIC 92], os agentes têm capacidades diferentes para resolver os problemas e podem ser classificados em: agentes reativos, muito simples e não inteligentes e os agentes cognitivos, inteligentes. Hoje, observamos que além 
destes, existem outros tipos de agentes como os agentes conversacionais, os softbots, os agentes pedagógicos, entre outros [JAQ 2001].

Neste trabalho, propõe-se o desenvolvimento e implementação de um personagem animado para um agente pedagógico animado: o agente mediador do ambiente educacional colaborativo MACES. Os agentes pedagógicos animados são um tipo especial de agente, que descrevermos mais sucintamente na próxima seção.

\section{Agentes Pedagógicos Animados}

Agentes pedagógicos são, segundo Giraffa [GIR 99], aqueles utilizados em sistemas que utilizam o paradigma de agentes desenvolvidos para fins educacionais, podendo atuar como tutores virtuais, estudantes virtuais, ou ainda companheiros virtuais de aprendizagem, tendo como objetivo auxiliar os estudantes no processo de ensinoaprendizagem.

Os agentes pedagógicos que utilizam-se de recursos multimídia para apresentar ao usuário um personagem animado que interaja com ele são conhecidos como agentes pedagógicos animados [JAQ 2001].

Segundo [JOH 2000], o agente pedagógico animado é o novo paradigma para ambientes de ensino, pois estes agentes podem demonstrar tarefas complexas, empregar locomoção e gestos para focar a atenção de alunos nos aspectos importantes das tarefas e expressar respostas emotivas para a situação de tutorial.

Eles são a grande promessa para a ampliação da comunicação num tutorial, incrementando habilidades no ambiente de ensino, prendendo a atenção e motivando o aluno.

Quando o agente está inserido em um ambiente virtual de simulação, o aluno pode aprender e praticar habilidades no mundo virtual. Com estes agentes, o computador pode interagir com os alunos através de iniciativa mista, diálogo tutorial no papel de professor ou companheiro aprendiz. Ele pode se comunicar de forma verbal e não verbal. O agente pode demonstrar uma ação enquanto fala. Ele pode usar a contemplação, afirmação com a cabeça, expressões faciais que podem ser um feedback nas declarações dos alunos, sem a necessidade da comunicação verbal e ruptura do raciocínio do aluno durante a realização da tarefa. Todo esse dispositivo não verbal são componentes naturais do diálogo humano.

$\mathrm{O}$ agente pedagógico animado apresenta as vantagens de aumentar a comunicação entre alunos e computadores, e incrementa a habilidade do computador para engajar e motivar o aluno.

Estes agentes pedagógicos compartilham aspectos em comum com agentes sintéticos desenvolvidos por aplicações de entretenimento. Eles devem dar ao usuário a impressão de serem naturais e confiáveis. Eles tornam a experiência de ensinar (aprender) mais agradável.

Alguns exemplos de agentes pedagógicos animados são:

$\sqrt{ }$ STEVE (Soar Training Expert for Virtual Environments) e ADELE (Agent for distance Learning: Light Editor) [JAQ 2000] [JAQ 2001] [BER 2001] [JOH 98] [JOH 99] [JOH 2000];

$\sqrt{ }$ Vincent (para sistemas de ensino-aprendizagem baseados na Web) [JAQ 2000] [JAQ 2001] [PAI 98]; 
$\sqrt{ }$ HERMAN THE BUG (inseto que habita o Design-A-Plant) e COSMO ajuda na solução de problemas no Internet Protocol Advisor [JAQ 2000] [JAQ 2001] [LES 96] [LES 98] [LES 98a] [LES 99] [JOH 2000];

$\sqrt{ }$ PPP Persona (guia os alunos através de material na Web) [AND 98] [AND 98a] [AND 98] [LES 2000].

Um agente animado pedagógico, com uma rica e interessante personalidade, pode fazer com que o aprender seja mais divertido. $\mathrm{O}$ aluno que interage com o agente pedagógico animado pode ter uma percepção mais positiva da experiência global da aprendizagem e pode optar por passar mais tempo no ambiente de ensino.

\section{Ambiente Educacional Colaborativo MACES}

O personagem animado, PAT, foi desenvolvido como a interface do agente pedagógico Mediador do ambiente MACES (Multiagent Architecture for an Collaborative Educational System) [AND 2001] [JUN 2001] [JAQ 2002]. MACES é um sistema educacional colaborativo à distância modelado segundo a abordagem multiagente apresentada nas seções 0 e 0 . Nesta seção, apresentamos a arquitetura do ambiente MACES.

O sistema proposto é composto por agentes humanos (usuários e tutores) e por cinco classes de agentes artificiais: o agente diagnóstico, o agente mediador, o agente colaborativo, o agente social e o agente semiótico. O agente diagnóstico implementa o conceito de zona de desenvolvimento proximal (ZDP) defendida por Vygotsky [VYG 84], visando transformar habilidades potenciais em habilidades reais e buscando expandir a capacidade de desenvolvimento sócio-cognitiva do aluno. $O$ agente colaborativo é responsável por mediar/monitorar a interação entre grupos de alunos em ferramentas síncronas de comunicação entre os alunos (por exemplo, Chat). $\mathrm{O}$ agente social deve estabelecer a integração da sociedade formando grupos de alunos para estudo e criando um agente colaborativo para cada grupo formado. O agente semiótico é responsável pela utilização de signos, conceitos e linguagem repassados ao agente mediador ou agente colaborativo e, conseqüentemente, ao aluno no processo de aprendizagem. $\mathrm{O}$ agente mediador auxilia no processo de internalização do aluno decorrente do contato com o ambiente social de EAD. Ele é responsável pela interface de comunicação do aluno com o ambiente, apresentando conteúdo pedagógicos e atividades e também por inferir os estados afetivos do aluno a fim de apresentar estratégias afetivas que visam promover no aluno um estado afetivo mais positivo para a aprendizagem. A fim de interagir afetivamente com o aluno, propõe-se a implementação de um personagem animado para este agente [JAQ 2003a] [JAQ 2003b]. Maiores detalhes do sistema se encontram descritos em [AND 2000], [AND 2001] e [JAQ 2002].

Os agentes artificiais monitoram e auxiliam os agentes humanos em suas atividades colaborativas. No sistema proposto, todos os personagens, usuários e agentes, são modelados como agentes sociais, integrados em um ambiente de aprendizagem colaborativa.

A ilustra a arquitetura do sistema proposto.

Os agentes diagnóstico, mediador e colaborativo são responsáveis por monitorar e interagir com os usuários e os agentes semiótico e social auxiliam nas atividades relacionadas a toda a sociedade. Assim, existe um agente diagnóstico e um agente mediador para cada usuário, um agente semiótico e um agente social para toda a 
sociedade, e um agente colaborativo para cada grupo de usuários formado com características em comum [JAQ 2002].

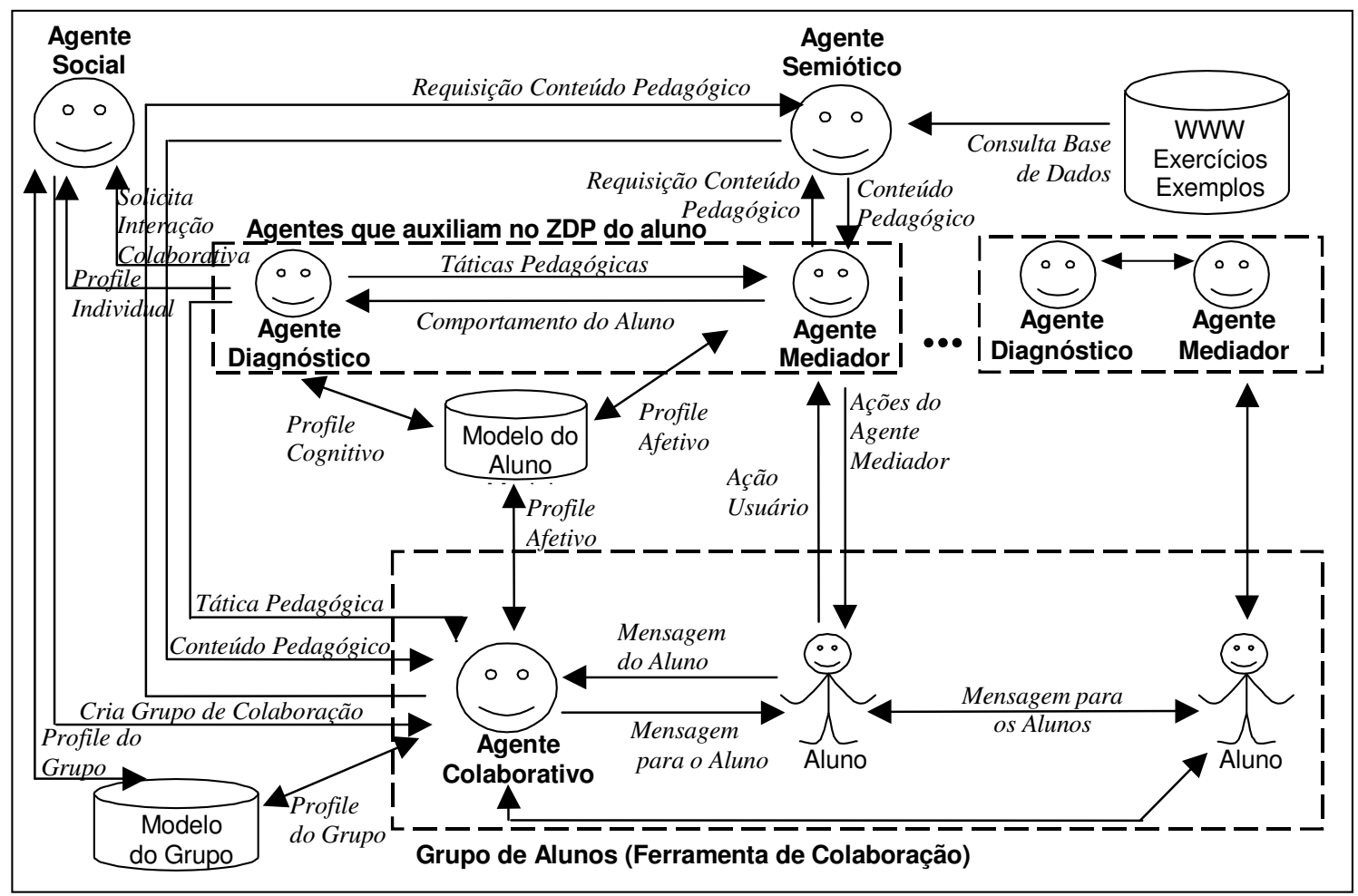

Figura 1. Sociedade de Agentes de Colaboração no Ambiente de Aprendizagem MACES [JAQ 2002]

Neste trabalho, propomos a implementação de um personagem animado (e sua arquitetura para a apresentação de comportamentos animados) para o agente mediador. A proposta deste trabalho encontra-se descrita na próxima seção. 


\section{$O$ personagem animado proposto}

Para a modelagem e implementação do personagem animado que compõe o agente proposto, foram realizadas algumas entrevistas com pedagogas, psicopedagogas e psicólogas, dos quais retirou-se as seguintes informações como características que um agente tutor deve possuir:

$\sqrt{ } \mathrm{O}$ personagem deve ser de corpo inteiro, homem ou mulher e de tamanho proporcional à tela, dando uma noção do todo, sendo mais próximo da realidade.

$\sqrt{ }$ Deve ter reações (expressões) faciais de alegria, tristeza, incentivo, apoio, espanto, admiração, calma, poderia pular, fazer careta, sentar e abaixar a cabeça, ficar de costas, tapar o rosto, ficar cabisbaixo, expressando emoções de acordo com o momento, fazendo com que o usuário reflita porque não está indo bem nos exercícios, sem ter de interromper o usuário com uma intervenção escrita ou falada.

$\sqrt{ }$ O personagem deve aparecer o tempo todo, interagir com o usuário e entrar em ação com a postura adequada ao momento do acontecimento, momento certo para responder com humor ou sério, com mensagens de incentivo, sem chamar demais a atenção para si. As mudanças de postura e face devem ser de forma sutil, não de forma brusca como os desenhos das faces de alegre e triste dos cartazes de empresas.

$\sqrt{ }$ Ele deve se mover na tela, gesticular, mexer a boca, os braços. Deve ter posturas diferentes em momentos diferentes, mas de forma descontraída para não tornar monótono o personagem. Deve usar roupas normais, descontraídas. Não muito formais. Deve ser colorido por ser mais estimulante, mas não cores muito apagadas como cinza e nem muito vivas como vermelho-sangue. Cores como azul, amarelo, rosa, verde. Ele deve ser um personagem mesclado, isto é, não muito certinho e nem muito atirado, não um personagem modelo, perfeito. Deve falar e escrever, usando o balão para comunicação escrita.

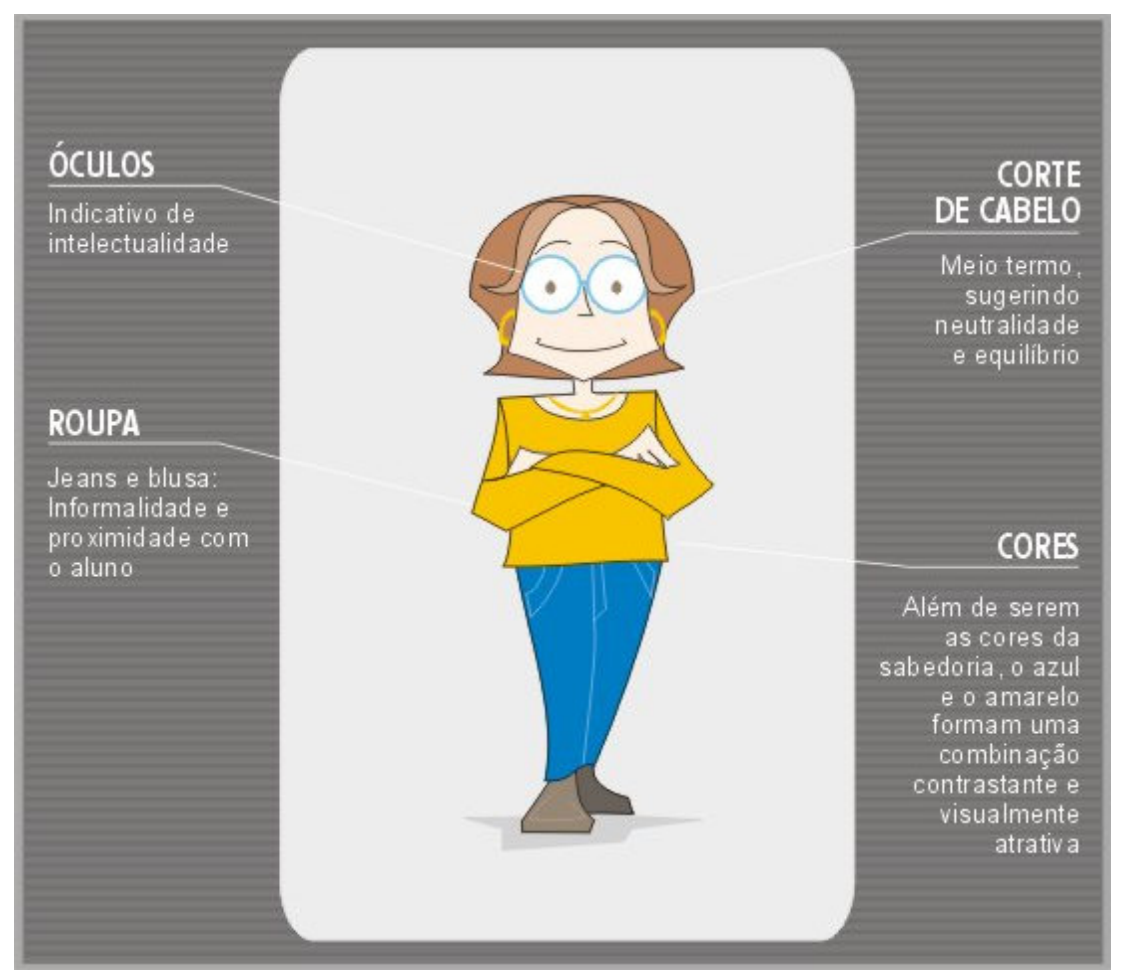

Figura 2. A Aparência do Personagem Animado PAT. 
$\sqrt{ }$ A estratégia para mudar o quadro afetivo pode ser, por exemplo, na terceira vez que o usuário errou, o personagem entra para dar umas dicas. Na quarta vez, ele faz uma careta e na quinta vez, ele poderia dar a resposta. Se o usuário estiver acertando tudo, ele deve interagir esporadicamente e de forma a reforçar a ação do usuário.

$\sqrt{ }$ O personagem poderia dar a opção de o usuário escolher, no início do programa, se quer que o personagem seja homem ou mulher, escolher a cor, etc.

$\mathrm{O}$ agente pedagógico animado deste trabalho está sendo desenhado com base nestes itens acima descritos. Na Figura 2, podemos observar a aparência do agente animado PAT.

\section{Arquitetura interna do personagem animado}

O personagem PAT foi implementado como interface para o agente Mediador (ver seção 3), um agente pedagógico animado do ambiente MACES. Para que PAT possa apresentar seus comportamentos animados, é necessário uma arquitetura que a permita escolher entre os comportamentos físicos e verbais disponíveis, aqueles que ela irá mostrar. Na Figura 3 observamos a arquitetura interna do personagem animado para apresentação dos comportamentos animados e afetivos. Esta arquitetura é formada pelo Módulo de Geração de Comportamentos.

O Módulo de geração de comportamentos (MGC) verbais e não-verbais afetivos é responsável por receber as táticas afetivas do Módulo de Raciocínio do Agente Mediador (Agente Mediador - tese de Patrícia Jaques [JAQ 2003]), que podem ser:

Uma tática pedagógica cognitiva como a apresentação de um exercício mais simples ou;

Comportamentos verbais e não-verbais afetivos: o MGC vai apenar tratar esse tipo de táticas.

O Módulo de geração de comportamentos verbais e não-verbais afetivos do personagem animado (PAT) é formado pelos seguintes componentes: seleção, montagem e apresentação. O personagem recebe do Módulo de Raciocínio do agente Mediador, através do Módulo de geração de comportamentos verbais e não-verbais afetivos, uma mensagem solicitando um comportamento animado físico e/ou verbal. $\mathrm{O}$ componente seleção escolhe aleatoriamente um comportamento do tipo solicitado junto com o comando para o Microsoft Agent fazer a animação na base de dados (BD) de comportamentos. Ele envia para o componente montagem que é responsável por gerar o código JavaScript e manda para o componente apresentação que gera a página html com o código JavaScript e o comando para o Microsoft Agent. Ele envia esta página HTML para o browser do usuário (usuário) que chama o Microsoft Agent e faz a animação solicitada. Todo esse módulo foi implementado como uma página JSP em Java que é executado no servidor e apenas mostrado no browser o usuário, ver Figura 3.

Para cada tipo de comportamento (por exemplo: comportamento encorajamento), existem várias animações diferentes que podem ser exibidas. Isso é implementado para que o personagem pareça mais credível, isto é, mais real [BAT 94]. Se o agente apresentasse sempre o mesmo comportamento para algumas situações, o agente pareceria monótono ao aluno. Como existe mais de uma animação possível para cada tipo de comportamento, o agente na hora da apresentação, deve escolher aleatoriamente uma destas animações para ser exibida. 
Cada um dos componentes do módulo de geração de comportamentos do personagem PAT encontra-se descrito a seguir.

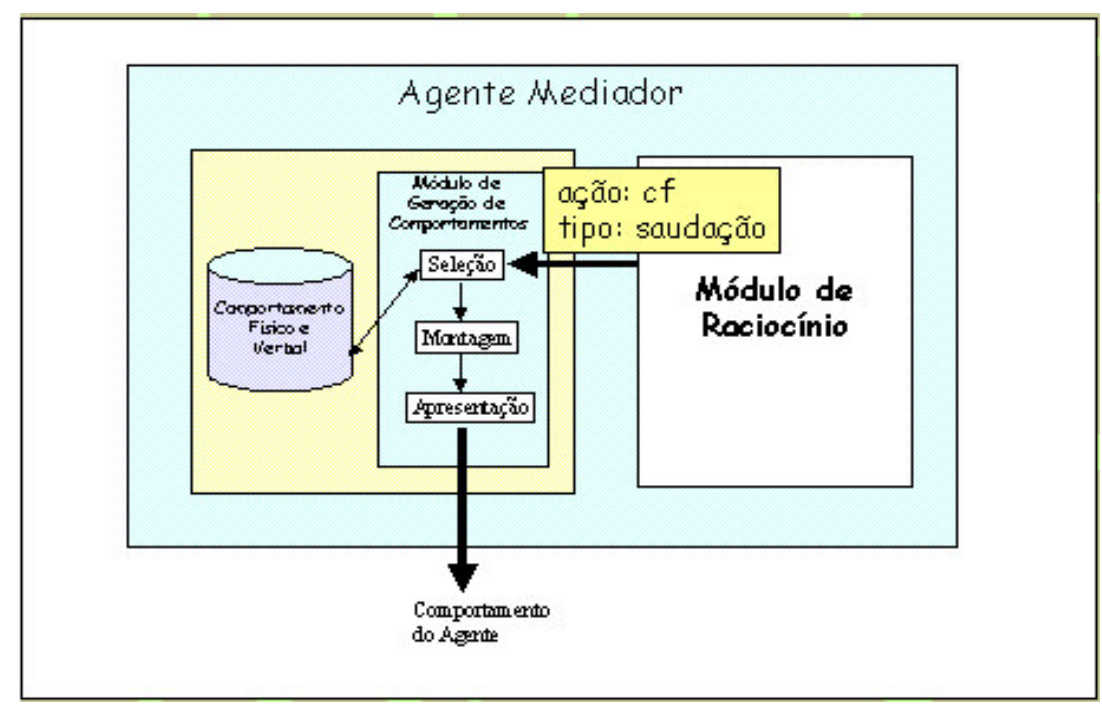

Figura 3. Arquitetura do Módulo de Geração de Comportamentos Verbais e Não-Verbais Afetivos

\section{Componente SELEÇÃO:}

O componente seleção é responsável por escolher um comportamento aleatório (físico ou verbal) do tipo solicitado pelo Módulo de Raciocínio.

Quando é escolhido um comportamento verbal, cv do campo ACAO, é devolvida uma fala, obtida do campo FALA, para o personagem. Quando é escolhido um comportamento físico, cf do campo ACAO, é devolvido um movimento, código do campo OPCIONAL, e uma fala, obtida do campo FALA, do comportamento verbal cujo campo TIPO tenha o mesmo valor do campo TIPO do comportamento físico (ver

\section{Tabela 1).}

Ele realiza uma escolha aleatória entre os comportamentos possíveis. A escolha aleatória destes comportamentos se dá da seguinte forma: o personagem grava no BD o horário em que aquele determinado comportamento foi exibido pela última vez. Quando o módulo de Raciocínio do agente Mediador solicitar um comportamento, o componente seleção vai escolher um dos comportamentos do tipo solicitado aleatoriamente, mas vai verificar no $\mathrm{BD}$ se aquele comportamento foi exibido por último, se sim, ele vai escolher outro comportamento e mostrar. O campo $\boldsymbol{L} \boldsymbol{R} \boldsymbol{U}$ do $B D$ contém um número indicando a ordenação dos registros segundo a sua utilização.

\section{Componente MONTAGEM:}

O componente montagem é responsável por gerar o código JavaScript para o Microsoft Agent movimentar o personagem.

Ele recebe do componente seleção um pedido de fala ou movimento, e lê na base de dados o código javascript que aciona a respectiva animação no Microsoft Agent. Após, envia esta informação ao componente apresentação. 


\section{Componente APRESENTAÇÃO:}

O componente apresentação gera a página HTML no browser do usuário. Esta página contém o código JavaScript (enviado pelo componente Montagem) que chama o Microsoft Agent para a movimentação do personagem.

Todo esse módulo foi implementado como uma página $\mathrm{JSP}^{1}$ em Java.

O código Java é executado no servidor e o browser do usuário recebe apenas o resultado, que é uma página HTML com o código JavaScript que aciona uma determinada animação do MSAgent instalado na máquina do usuário.

\section{A Base de Dados}

Os comportamentos físico e verbal são armazenados em um banco de dados (BD) HSQLDB (freeware), como mostra a

\section{Tabela 1.}

Para a melhor manipulação dos dados desta tabela, foram implementadas interfaces (desenvolvidas em JSP) que permitem a visualização, modificação e exclusão das linhas desta tabela.

A coluna ID contém um identificador único para as ações do agente. Este campo é usado como chave da tabela. A coluna ACAO contém o tipo da ação. Eles podem ser comportamentos verbais ou comportamentos físicos. Os comportamentos verbais são representados pelo símbolo $\mathrm{CV}$ e contém as falas do agente. Os comportamentos físicos (CF) contêm as animações que o agente pode exibir. No campo DESCRIÇÃO é apresentada uma descrição deste comportamento. O campo FALA contém a fala a ser dita pelo agente. Para as falas, usamos o sintetizador de voz da Microsoft. O campo OPCIONAL contém o código JavaScript que o agente deve escrever na página HTML para o Microsoft Agent gerar o comportamento solicitado. O campo LRW é usado para controlar que não sejam exibidos comportamentos repetitivos. A

Tabela 1 apresenta alguns exemplos de comportamentos que serão exibidos pelo agente. Estes comportamentos, táticas pedagógicas afetivas do agente Mediador, foram definidas no trabalho de tese da Patrícia Jaques [JAQ 2003].

Tabela 1. Banco de Dados de Comportamentos Físicos e Verbais

\begin{tabular}{|c|c|c|c|c|c|}
\hline $\mathrm{D}$ & $\mathrm{CAO}^{\mathrm{A}} \mathrm{O}$ & DESCRICAO & FALA & $\begin{array}{r}\text { OP } \\
\text { CIONAL }\end{array}$ & RU \\
\hline & 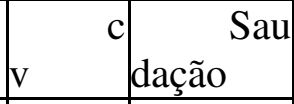 & $\begin{array}{l}\text { Comportamento de } \\
\text { Saudação }\end{array}$ & Olá！ & & 4 \\
\hline & c $\left.\right|_{\text {dação }} \mathrm{Sau}$ & $\begin{array}{l}\text { Comportamento de } \\
\text { Saudação } \\
\end{array}$ & Bom Dia! & & 1 \\
\hline & $\left.\mathrm{c}\right|_{\text {dação }} \mathrm{Sau}$ & $\begin{array}{l}\text { Comportamento de } \\
\text { Saudação }\end{array}$ & Boa Tarde! & & 5 \\
\hline & $\left.\mathrm{c}\right|_{\mathrm{da}} \quad \mathrm{Aju}$ & $\begin{array}{l}\text { Agente oferencendo ajuda } \\
\text { ao aluno }\end{array}$ & $\begin{array}{l}\text { Precisa de } \\
\text { ajuda? }\end{array}$ & & 2 \\
\hline
\end{tabular}

1 JSP é uma página HTML que contém código Java. 


\begin{tabular}{|c|c|c|c|c|c|c|}
\hline & $\mathrm{c}$ & da & Agente ajuda aluno & $\begin{array}{l}\quad \text { Posso olhar } \\
\text { o exercício para te } \\
\text { ajudar?? }\end{array}$ & & 7 \\
\hline & $\mathrm{c}$ & da & Comportamento de ajuda & \begin{tabular}{l}
\multicolumn{1}{c}{ Está no } \\
caminho certo, \\
mas dê uma \\
olhada no capítulo \\
$3 ! !$
\end{tabular} & & 6 \\
\hline & $\mathrm{c}$ & posta & $\begin{array}{l}\text { Comportamento de } \\
\text { resposta }\end{array}$ & $\begin{array}{l}\text { Achei a } \\
\text { resposta neste } \\
\text { livro aqui! } \\
\end{array}$ & & 15 \\
\hline & $\mathrm{c}$ & posta & $\begin{array}{l}\text { Comportamento de } \\
\text { resposta }\end{array}$ & $\begin{array}{l}\text { Depois que } \\
\text { li o capítulo } 3 \\
\text { descobri que a } \\
\text { resposta é... }\end{array}$ & & 21 \\
\hline 0 & $\mathrm{c}$ & posta & $\begin{array}{l}\text { Comportamento de } \\
\text { Resposta. }\end{array}$ & $\begin{array}{r}\text { A resposta a } \\
\text { sua pergunta é: }\end{array}$ & & 20 \\
\hline 1 & $\mathrm{c}$ & posta & $\begin{array}{l}\text { Comportamento de } \\
\text { resposta }\end{array}$ & $\begin{array}{l}\text { A resposta } \\
\text { certa é: }\end{array}$ & & 17 \\
\hline 2 & $\mathrm{c}$ & dação & Agente abana. & Saudação & dação & 0 \\
\hline 4 & $\mathrm{c}$ & da $\quad$ Aju & $\begin{array}{l}\text { pega uma lupa e faz } \\
\text { movimento de procura. }\end{array}$ & Ajuda & da $\quad$ Aju & 8 \\
\hline 8 & $\mathrm{f}$ & oria & $\begin{array}{l}\text { Agente se inclina } \mathrm{p} / \\
\text { frente, aumenta o temanho da } \\
\text { boca e diz Uh! }\end{array}$ & Tutoria & $\begin{array}{r}\text { Get } \\
\text { Attention }\end{array}$ & 0 \\
\hline 9 & $\mathrm{c}$ & oria & $\begin{array}{l}\text { Agente aparece com o } \\
\text { livro e esconde atrás dele. }\end{array}$ & Tutoria & $\begin{array}{r}\text { Writ } \\
\text { eReturn }\end{array}$ & 8 \\
\hline 0 & $\mathrm{c}$ & posta ${ }^{\text {Res }}$ & $\begin{array}{l}\text { Agente pega um livro na } \\
\text { estante, lê e fala. }\end{array}$ & Resposta & \begin{tabular}{|l|} 
Res \\
posta:
\end{tabular} & 6 \\
\hline
\end{tabular}

\section{Conclusões e Trabalhos Futuros}

Temos observado que pesquisadores em educação à distância têm apostado no uso de agentes animados como softwares educacionais [GIR 99] [JAQ 2001] devido a vantagens como demonstrar tarefas, empregar gestos e locomoção para focar a atenção dos alunos e expressa respostas emotivas para a situação de um tutorial, o que permite a estes agentes a ampliação da comunicação, prendendo a atenção e motivando o aluno num tutorial [JOHN 2000].

Com estes agentes, o aluno pode aprender e praticar habilidades no mundo virtual e o computador pode interagir com os alunos através de diálogo tutorial no papel de professor ou companheiro aprendiz.

$\mathrm{O}$ agente pedagógico animado apresenta as vantagens de aumentar a comunicação entre alunos e computadores, e incrementa a habilidade do computador para engajar e motivar o aluno. 
Um agente pedagógico animado, com uma rica e interessante personalidade, pode fazer com que o aprender seja mais divertido. $\mathrm{O}$ aluno que interage com o agente pedagógico animado pode ter uma percepção mais positiva da experiência de aprendizagem e pode optar por passar mais tempo no ambiente de ensino. Ele pode se comunicar de forma verbal e não verbal. Pode demonstrar uma ação enquanto fala, pode usar a contemplação, afirmação com a cabeça, expressões faciais que podem ser um feedback nas declarações dos alunos, sem a necessidade da comunicação verbal e ruptura do raciocínio do aluno durante a realização da tarefa.

Todo esse dispositivo não verbal são componentes naturais do diálogo humano.

O grupo GIA - UFRGS observou que essas vantagens seriam também interessantes quando considerando as emoções do aluno na interação tutor-aluno [JAQ 2003a]. Assim, este trabalho vem propor desenvolver a interface de um agente animado que está inserido em um ambiente computacional de ensino: o MACES. Para definir a aparência do personagem foram realizadas entrevistas com educadores, psicólogos, pedagogas, designers para parecer mais próximo da realidade de se conseguir colocar emoção no computador e fazer com que esta ferramenta mais a Web fique como uma alternativa à educação para todos.

Procurou-se construir personagem PAT de forma que ele pareça credível [BAT 94] e o se divirta com o mesmo, no curso que escolher fazer via Web (com o MACES), e que o usuário se identifique com ele assim como acontece nos cursos presenciais. $\mathrm{O}$ personagem tenta auxiliar o estudante de uma forma descontraída, não muito formal, mas de forma a ter credibilidade.

A validação de PAT será feita na tese de doutorado de Patrícia Jaques, que desenvolve o Agente Mediador. 


\section{Referências Bibliográficas}

[AND 98] ANDRÉ, Elizabeth et al. Employing AI Methods to Control the Behavior os Animated Interface Agents. 1998. Disponível em: $<$ http://www.ai.univie.ac.at/ paolo/Iva/vu-sa2000/os/andre_etal_aaij.os.gz>. Acesso em jun. 2002.

[AND 98a] ANDRÉ, Elizabeth et al. WebPersona: A Life-Like Presentation Agent for the Worl-Wide Web. 1998. Disponível em: $<$ http://www.dfki.de/imedia/papers/kbs.ps.gz >. Acesso em jun. 2002.

[AND 2000] ANDRADE, Adja F. et al. A Computational Model of Distance Learning Based on Vygotsky's Socio-Cultural Approach. Anais... Workshop de Ambientes de Aprendizagem baseados em Agentes. XI Simpósio Brasileiro de Informática na Educação. Maceió, 8 a 10 de Novembro de 2000.

[AND 2001]ANDRADE, Adja F. et al. Uma Proposta de Modelo Computacional de Aprendizagem à Distância Baseada na Concepção Sócio-Interacionista de Vygotsky. Anais... MABLE WORKSHOP (Multi-Agent Based Learning Environments). X International Conference on Artificial Intelligence on Education. Antonio, Texas, May 19-23 2001.

[BAT 94] BATES, J. The Role of Emotion in Believable Agents. Communications of ACM, 37(7):122-125, 1994.

[BER 2001] BERCHT, Magda. Em Direção a Agentes Pedagógicos com Dimensões Afetivas. 2001. 152p. Tese( Doutorado em Ciência da Computação) Instituto de Informática, UFRGS, Porto Alegre.

[BIC 99] BICA, Francine. Eletrotutor III - Uma Abordagem Multiagente para o Ensino à Distância. 1999. Dissertação (Mestrado em Ciência da Computação) Instituto de Informática, UFRGS, Porto Alegre.

[GIR 99] GIRAFFA, Lúcia M. M. Uma arquitetura de tutor utilizando estados mentais. Tese (Doutorado em Ciências da Computação) - Instituto de Informática, UFRGS, Porto Alegre. 1999.

[JAQ 2000] JAQUES, Patrícia A. et al. Agentes Pedagógicos Animados. Porto Alegre: PPGC da UFRGS, 2000.

[JAQ 2001] JAQUES, Patrícia et al. Interação com Agentes Pedagógicos Animados: Um Estudo Comparativo. In: WORKSHOP SOBRE FATORES HUMANOS EM SISTEMAS COMPUTACIONAIS, IHC, 4., 2001, Florianópolis, SC. Anais... Florianópolis: UFSC, 2001.

[JAQ 2002] JAQUES, Patrícia A.; JUNG, João L.; ANDRADE, Adja F. de; BORDINI, Rafael H.; VICARI, Rosa M.. Using Pedagogical Agents to Support Collaborative Distance Learning in Computer Supported Collaborative Learning Proceedings of CSCL 2002. New Jersey: Lawrence Erlbaum Associeates, 2002. pp. 546-547.

[JAQ 2003a] JAQUES, Patricia A.; PESTY, Sylvie; VICARI, Rosa. An Animated Pedagogical Agent that Interacts Affectively with the Student. 11th International Conference on Artificial Intelligence in Education. Proceedings... Sydney, Australia, 2003.

[JAQ 2003b] JAQUES, Patricia A.; BERCHT, Magda; BOCCA, Everton; VICCARI, Rosa. Cognitive Reasoning to Respond Affectively to the Student. In: The 
IASTED International Conference on Computers and Advanced Technology in Education. Proceeedings... June 30 - July 2, 2003. Rhodes, Greece.

[JEN 98] JENNINGS, Nicholas R. et al. A Roadmap of Agent Research and Development. Autonomous Agents and Multi-Agent Systems. 1, 275-306 -Kluwer Academic Publishers, Boston:1998.

[JOH 98] JOHNSON, L. et al. Pedagogical Agentes on The Web. In: ITS'98 Workshop on Pedagogical agents, 4,1998. San Antônio. Anais. San Antonio:1998.p.2-7. Também disponivel na WWW em http://www.isi.edu/isd/ADE/papers/its98/ITS98-WW.htm.

[JOH 99] JOHNSON, L. et al. Pedagogical Agents on The Web. Disponível na WWW em http://www.isi.edu/isd/ADE/papers/agents99/agents99.htm

[JOH 2000] JOHNSON, Lewis; RICKEL, Jeff; LESTER, James. Animated Pedagocial Agents: Face-to-Face Interaction in Interactive Learning Environments. International Journal of Artificial Intelligence in Education, 11, pp. 47-78. Disponível na WWW em http://www.csc.ncsu.edu/eos/users/l/lester/www/imedia/apa-ijaied2000.html acessado em novembro de 2001.

[JUN 2001] JUNG, João Luiz. Concepção e Implementação de um agente Semiótico como Parte de um Modelo Social de Aprendizagem à Distância. Dissertação de Mestrado. Porto Alegre. Instituto de Informática da UFRGS, 2001.

[LES 96] LESTER, James C. et al. Cosmo: A Life-like Animated Pedagogical Agent with Deictic Believability. Disponível na WWW em http://www.csc.ncsu.Edu/eos/users/l/lester/www/imedia/papers.html acessado em 2000.

[LES 98] LESTER, James C. et al Visual Emotive communication in Lifelike Pedagogical Agents. Disponível na WWW em http://wwwcsc.ncsu.edu/eos/users///lester/Public/ipa-its-98.ps acessado em junho de 2002.

[LES 98a] LESTER, James C. et al. Deictic Believability: Coordinated Gesture, Locomotion, and Speech in Lifelike Pedagogical Agents. Disponível na WWW em http://www.csc.ncsu.edu/eos/users///lester/Public/cosmo-aai-98.ps acessado em junho 2002.

[LES 99] LESTER, James C. et al. Achieving Affective Impact: Visual Emotive Communication in Lifelike Pedagogical Agents. International Journal of Intelligence in Education, 1999, 10, 278-291.

[LES 2000] LESTER, James C. et al. Animated Pedagogical Agents: Face-toFace Interation in Learning Environments. Disponível por WWW em http://www.csc.ncsu.edu/eos/users/l/lester/www/imedia/apa-ijaied-2000.html acessado em Novembro de 2001.

[LIE 2002] LIEBERMAN, Henry. Autonomous Interface Agents. Media Laboratory. Massachusetts Institute of Technology. USA. Disponível por WWW em http://www.media.mit.edu/ lieber .

[MIC 2003] MICROSOFT

http://www.microsoft.com/msagent/.

CORPORATION. Microsoft Agent.

[PAI 98]PAIVA, A. et al. Vincent, an Autonomous Pedagogical Agent for Onthe-Job Training. In: ITS'98, LNCS 1452, pp.584-593, 1998. Ed. V. Shute Pub. Springer-Verlag 1998.

[SIC 92] SICHMAN, J. et al. When can Knowledge-based Systems be called Agents? In: SIMPÓSIO BRASILEIRO DE INTELIGÊNCIA ARTIFICIAL, 9., 1992, Rio de Janeiro. Anais... Rio de Janeiro: SBC, 1992.p.172-185. 
[VYG 84] VYGOTSKY, L. A formação social da mente. São Paulo : Martins Fontes, 1984.

[WOO 95] WOOLDRIDGE, $M$. et al. Intelligent Agents: Theory and Practice. Knowledge Engineering Review, [S.1.], v.10, n.2, p.115-152, 1995. Disponível na WWW em : http://www.elec.qmw.ac.uk/daí/pubs acessado em Janeiro 2003. 